\title{
Preface to SPECIAL ISSUE ON MOdeRn INTELLIGeNt SYSTEMS CONCEPTS ॥
}

DOI: 10.14313/JAMRIS/4-2020/42

Over these last decades, technological advancements have broken their records. We are witnessing an unprecedented technological revolution. The use of Intelligent Systems, IoT, Cloud Computing, Big Data, and more particularly Artificial Intelligence including Deep Learning and Machine Learning are becoming the norm in almost all activities. These disciplines are seeing their applications developed in all sectors including education, industry, health, agriculture, economy, finance, energy, environment, security, transport, societal challenges, and in general, in any field that one can think of. Our goal, as researchers, is to guide all these proposed disciplines to streamline and facilitate human daily activities.

This JAMRIS special issue offers a variety of ideas discussing different subjects having as common denominator important technical proposals to try to contribute to the evolutions in intelligent systems. The papers included in this session are selected from the International Conference on Modern Intelligent Systems Concepts, which was held in Morocco in December 2018 (MISC'2018). In this special issue, six papers are presented.

In the first paper, K. Ounachad, M. Oualla, A. Sadiq and A. Souhar propose a novel method for human Face Emotion Recognition (FER). FER systems aim to recognize a face emotion in a dataset. The authors explain how they generate seven referential faces suitable for each kind of facial emotion based on perfect face ratios and five classical averages: arithmetic mean, geometric mean, harmonic mean, contraharmonic mean and quadratic mean. The authors' idea is to extract perfect face ratios for emotional face and for each referential face as features, they calculate the distance between them using fuzzy hamming distance. To do so, they used the point landmarks in the face and sixteen features based on the distance between different portions of the perfect face. The authors concluded that their method gives the very promising results.

In the second paper, A. Atassi and I. El Azami propose a hybrid deep learning system based on a combining between Gated Recurrent Unit (GRU) and Convolutional Neural Network (CNN). Firstly, they studied a system based on CNN. Then, they compare it with a second system that uses GRU. This hybrid system takes into account the positive points of the two previous systems, and takes the right choice of hyper-parameters recommended by the authors of both systems. In addition, the authors propose an approach to apply their system to the dataset of different languages, as used particularly in socials networks.

As for the third paper, A. Riadsolh, I. Lasri and M. ElBelkacemi tackle the problem of measuring customer' satisfaction in the banking sector using Naïve Bayes and Stanford NLP based on sentiment analysis. The authors propose and evaluate a real-time processing pipeline using the open-source tools in Microsoft Azure. They used Apache Kafka as data ingestion system, Apache Spark as a real-time data processing system, Apache Hbase for persistent distributed storage, and ElasticSearch and Kibana for visualization.

In their paper, M. Es-Sadqi, A. Idrissi and A. Benhassine presented different methods for solving optimization problems of redundancy in multi-state systems. Those methods are based on genetic algorithm and constraint satisfaction methods including Forward Checking algorithm. The authors propose an extension of the Forward checking algorithm. This last gave the best results in the comparative case studied, while allowing also the verification of the results of the cost and availability obtained. The configurations obtained are simple and homogeneous and rarely varied. The authors states that the orientation of the selection according to the application is a strong point of those methods. Their work has also helped not only proving that constraints based research improves complexity but also allows finding various and high quality solutions.

The authors M. Riyad, M. Khalil and A. Adib tackle the Convolutional Neural Networks (CNN) applied to Brain Computer Interface (BCI). A BCI is an instrument capable of commanding machine with brain signal. They propose an EEG classification system applied to BCI using the CNN for P300 problem. The system consists of three stages. The first stage is a Spatiotemporal convolutional layer which is a succession of temporal and spatial convolutions. The second stage contains 5 standard convolutional layers. Finally, a logistic regression is applied to classify the input EEG signal. The authors justify experimentally the necessity of a deep neural network rather than a shallow neural network. They state that their model allows the visualizing of the learned features and finally show that their approach is outperforming the existing models.

The last and not the least paper authored by K. Mrhar, O. Douimi and M. Abik, deals with the important rate of dropout in MOOCs. This high dropout rate in MOOCs can be related to diverse aspects, like the motivation of the learners, their expectations and the lack of social interactions. To tackle this problem, the authors present a dropout 
predictor model based on a neural network algorithm and sentiment analysis feature using the clickstream log and forum post data. Thus, they choose nine features that are the most correlated with learner's dropout. Then, they construct an artificial neural network ANN model to predict if the learner will dropout the next week. According to their study, they conclude that the neural network is more preferment than other baseline algorithm, including KNN, SVM and Decision Tree.

We consider that this special session presents some real advances in the field of Intelligent Systems. It contributes to its evolution, its development, its emergence, and particularly in its orientation and good practice in the service of the humanity.

We would like to thank all the authors for their interactions and interesting contributions as well as all the reviewers for their time, advices and suggestions.

In addition, we will not close this preface without warmly acknowledging the great efforts of the Editors, especially Professor Janusz Kacprzyk and the Managing Editor Katarzyna Rzeplinska-Rykala for their great help and support and to any person whom contribute to promote the International Journal JAMRIS.

\section{Editor:}

\section{Abdellah Idrissi}

MISC'2018 General Chair

Artificial Intelligence Group,

Intelligent Processing Systems Team (IPSS),

Computer Science Laboratory (LRI),

Computer Science Department,

Faculty of Sciences

Mohammed V University in Rabat, Morocco

email: idrissi@fsr.ac.ma,idrissi@ieee.org 